\title{
Middleware for heterogeneous subsystems interoperability in intelligent buildings
}

\begin{abstract}
Managing heterogeneous subsystems in an intelligent building has always been elusive due to lack of interoperability factor. This is due to the nature of heterogeneous systems in an intelligent building as a distributed entity that needs a certain degree of interoperability and joint execution of tasks for managing heterogeneous systems that consist of different platforms and protocols. There is a need for interoperability in an intelligent building with a bespoke solution that could make the heterogeneous subsystems 'talk' with each other and interoperate regardless of the operating platform. To solve the interoperability problem, Web Services technology is seen as a potential solution that could deal with the dynamic environment of an intelligent building. In this paper, we describe a SOAP-based middleware implementation for managing heterogeneous subsystems in an intelligent building. The proposed middleware solution utilizes the SOAP technology together with ECA rule mechanism in managing subsystems interoperation in a federated manner. Experiments and performance evaluation of the middleware had been carried out to justify the performance requirement of heterogeneous systems in an intelligent building.
\end{abstract}

Keyword: SOAP; Intelligent building; ECA; Web Services; Interoperability; Middleware 